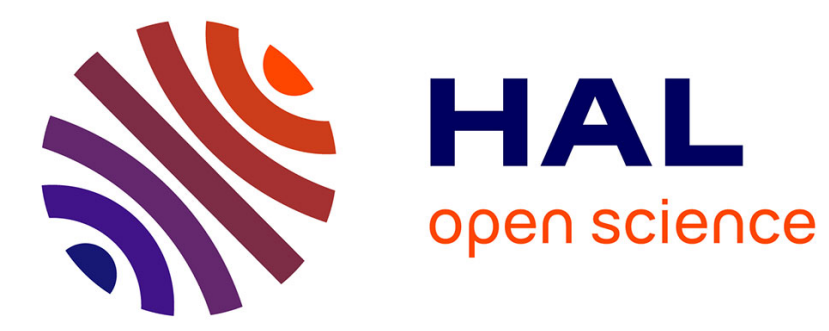

\title{
Examen de matériaux en vue de la récupération de chaleur par voie thermoélectrique
}

\author{
Jean Mahenc, René Routie
}

\section{To cite this version:}

Jean Mahenc, René Routie. Examen de matériaux en vue de la récupération de chaleur par voie thermoélectrique. Revue de Physique Appliquée, 1970, 5 (4), pp.653-658. 10.1051/rphysap:0197000504065300 . jpa-00243441

HAL Id: jpa-00243441

https://hal.science/jpa-00243441

Submitted on 1 Jan 1970

HAL is a multi-disciplinary open access archive for the deposit and dissemination of scientific research documents, whether they are published or not. The documents may come from teaching and research institutions in France or abroad, or from public or private research centers.
L'archive ouverte pluridisciplinaire HAL, est destinée au dépôt et à la diffusion de documents scientifiques de niveau recherche, publiés ou non, émanant des établissements d'enseignement et de recherche français ou étrangers, des laboratoires publics ou privés. 


\title{
EXAMEN DE MATÉRIAUX EN VUE DE LA RÉCUPÉRATION DE CHALEUR PAR VOIE THERMOÉLECTRIQUE
}

\author{
Par Jean MAHENC et René ROUTIE \\ Institut du Génie Chimique et Laboratoire d'Electrochimie de la Faculté des Sciences de Toulouse, \\ 118, route de Narbonne, 31, Toulouse, 04
}

(Reçu le 16 mars 1970)

\begin{abstract}
Résumé. - L'examen des propriétés thermoélectriques de matériaux tels que les chalcogénures métalliques est entrepris, en vue de leur utilisation possible en tant que thermoéléments. La variation de la concentration électronique de ces produits est assurée à l'aide d'un dopage électrochimique. Les facteurs de mérite sont calculés et la réalisation d'un élément de thermopile suggère l'orientation de l'effort technologique.
\end{abstract}

Abstract. - Thermoelectric properties of metallic chalcogenides are determined in order to use such materials in thermocells. An electrochemical doping is involved to vary the electronic concentration of those materials. The figure of merit are calculated and experiments with a thermocell suggest technical improvements.

Introduction. - Les chalcogénures métalliques offrent une grande variété de solides semi-conducteurs dont les propriétés thermoélectriques sont étroitement liées à l'indice stœchiométrique du composé $[1,2]$. Un examen systématique du pouvoir thermoélectrique, de la conductivité électrique et de la conductivité thermique de ces composés est rendu possible par la mise au point d'une technique électrochimique de dopage. La théorie statistique de FermiDirac donne un support théorique consistant à l'ensemble et autorise une exploitation féconde des résultats expérimentaux. Enfin, la réalisation de thermoéléments à partir de poudres polycristallines fortement comprimées ouvre la voie à la construction de dispositifs plus puissants.

I. Analyse théorique. - I. 1 COMPOSITION DES CHALCOGÉNURES MÉTALLIQUES. - La grande majorité des chalcogénures métalliques présentent des écarts, plus ou moins importants, par rapport à la stœchiométrie idéale.

L'emploi de piles solides, de chaînes symboliques suivantes $[3,4,5]$

$$
\begin{array}{ll}
\mathrm{Ag}-\mathrm{AgI}-\mathrm{Ag}_{x} \mathrm{~S}-\mathrm{Pt} & (\mathrm{P} 1) \\
\mathrm{Cu}-\mathrm{CuI}-\mathrm{Cu}_{x} \mathrm{~S}-\mathrm{Pt} & \text { (P 2) } \\
\mathrm{Cu}-\mathrm{CuI}-\mathrm{Cu}_{x} \mathrm{Se}-\mathrm{Pt} & \text { (P 3) } \\
\mathrm{Cu}-\mathrm{CuI}-\mathrm{Cu}_{x} \mathrm{Te}-\mathrm{Pt} & (\mathrm{P} \text { 4) }
\end{array}
$$

permet de déterminer avec précision la composition, définie par l'indice stœchiométrique $x$, des chalcogénures ci-dessus, à savoir : les sulfures d'argent et de cuivre, le séléniure et le tellurure de cuivre.

En effet, la force électromotrice $U$ de telles piles est une fonction du potentiel chimique du métal (argent ou cuivre) contenu dans le chalcogénure. Ainsi, à chaque valeur de l'indice stœchiométrique correspond une valeur du potentiel chimique du métal et par suite une valeur bien définie de la force électromotrice de pile. La connaissance de la caractéristique (force électromotrice-indice stœchiométrique) permet donc, par un simple repérage de force électromotrice, la détermination précise de la composition de l'élément considéré.

Les compositions limites de ces différents chalcogénures sont indiquées dans le tableau I.

\section{Tableau I}

Limites de composition des chalcogénures de $\mathrm{Cu}$ et $\mathrm{Ag}$

$\begin{array}{lccc}\begin{array}{c}\text { Chalco- } \\ \text { génure }\end{array} & \begin{array}{c}\text { Tempéra- } \\ \text { ture } \\ { }^{\circ} \mathrm{C}\end{array} & \begin{array}{c}\text { Indice } \\ \text { stœchiomé- } \\ \text { trique } \\ \text { maximum }\end{array} & \begin{array}{c}\text { Indice } \\ \text { stœchiomé- } \\ \text { métrique } \\ \text { minimum }\end{array} \\ - & - & - & - \\ \mathrm{Ag}_{2} \mathrm{~S} & 200 & 2,0020 & 2,0000 \\ \mathrm{Ag}_{2} \mathrm{~S} & 300 & 2,0025 & 2,0000 \\ \mathrm{Cu}_{2} \mathrm{~S} & 400 & 2,00 & 1,75 \\ \mathrm{Cu}_{2} \mathrm{Se} & 400 & 2,00 & 1,86 \\ \mathrm{Cu}_{2} \mathrm{Te} & 400 & 2,00 & 1,33\end{array}$

I. 2 CARACtères SEMI-CONDUCTEURS. - L'interprétation du mécanisme de la diffusion $[3,6]$ de l'argent dans son sulfure permet d'énoncer que l'argent excédentaire présent doit être considéré comme l'association d'ions $\mathrm{Ag}^{+}$et d'électrons. De même, le déficit en cuivre dans les chalcogénures cuivreux implique, 
pour satisfaire à la condition d'équilibre électrique interne, la formation de trous électroniques positifs.

C'est à la présence, dans ces matériaux, d'un gaz de charges électriques (électrons ou trous positifs) que doit être attirbué le caractère semi-conducteur de ces éléments.

La concentration $n$ des charges électriques est, dans le cas présent, reliée directement à l'indice stoechiométrique par la relation :

$$
n=(2 \pm x) \frac{N_{0}}{V_{\mathrm{m}}}
$$

où $N_{\mathrm{o}}$ est le nombre d'AVOGADRO; $V_{\mathrm{m}}$ est le volume molaire de l'élément considéré ; le signe + doit être attribué au sulfure d'argent (semi-conducteur de type $n$ ) et le signe - à tous les autres composés (type $p$ ).

D'autre part, la probabilité $F_{1 / 2}(\mu / R T)$ de rencontrer des charges dans la bande de conduction est donnée par :

$$
n=4 \pi \frac{\left(2 m^{*} k T\right)^{3 / 2}}{h^{3}} \cdot F_{1 / 2}\left(\frac{\mu}{R T}\right)
$$

où $m^{*}$ est la masse électronique effective des charges ; $k, h$ et $R$ sont respectivement les constantes de Boltzmann, de Planck et des gaz parfaits ; $T$ est la température absolue ; $F_{1 / 2}(\mu / R T)$ est la fonction de FermiDirac dont la connaissance permet de déterminer le potentiel chimique $(\mu / R T)$ des charges électriques [7].

On dispose donc, dans le cas présent, de matériaux semi-conducteurs dont la concentration électronique, le potentiel chimique électronique ainsi que la fonction de Fermi-Dirac correspondante peuvent être déterminés directement par la connaissance de l'indice stœchiométrique. Rappelons que cet indice stœechio- métrique peut être connu, par voie électrochimique, à l'aide de la force électromotrice des piles $\left(\begin{array}{ll}P & 1\end{array}\right)$ à (P 4).

I.3 PropriétÉS THERMó́LECTRIQUES. - L'étude théorique [8] de la conversion d'énergie thermique en énergie électrique fait intervenir, dans l'expression $\mathrm{du}$ rendement énergétique, à côté du rendement de Carnot, l'influence d'un facteur de qualité propre au matériau considéré. Désigné sous le nom de «facteur de mérite ", son expression est donnée par la relation :

$$
Z=\frac{\alpha^{2} \sigma}{K}
$$

où $\alpha$ est le pouvoir thermoélectrique (effet Seebeck) du matériau, $\sigma$ est la conductivité électrique et $K$ la conductivité thermique.

La connaissance de ce facteur de mérite nécessite donc l'étude préalable du pouvoir thermoélectrique et des conductivités électriques et thermiques, propriétés que l'on désigne sous le nom de propriétés thermoélectriques. Le tableau II résume les valeurs théoriques de ces différentes propriétés, telles qu'elles sont déduites de la statistique de Fermi-Dirac, appliquée au gaz électronique [8].

Les différents symboles figurant dans les formules du tableau II ont été précédemment définis, à l'exception de $e$ qui est la charge de l'électron, de $u$ qui désigne la mobilité électronique et de $r$ qui représente le caractère des forces de liaison, intervenant dans la relation entre le libre parcours moyen $l$ des électrons et leur énergie cinétique $\varepsilon$, soit $l=l_{0} \cdot \varepsilon^{r}$. Pour les réseaux ioniques (cas des chalcogénures) $r$ est estimé égal à 0,5 .

Les différentes fonctions de Fermi-Dirac ( $\mathrm{Fr}$,

\section{TABLEAU II}

\section{Expressions des propriétés thermoélectriques}

Propriété thermoélectrique

Pouvoir thermoélectrique

Conductivité électrique

Conductivité thermique électronique
Formule correspondante

$$
\alpha= \pm \frac{k}{e}\left[\frac{r+2}{r+1} \cdot \frac{F_{1 / 2}}{F_{3 / 2}}-\frac{\mu}{R T}\right]
$$

$$
\alpha= \pm \frac{k}{e}\left[r+2-\frac{\mu}{R T}\right] \text { (gaz non dégénéré) }
$$$$
\sigma=n . e . u
$$

$$
\sigma=\frac{16 \pi m^{*} e^{2}}{3 h^{3}} \cdot l_{0} \cdot(K T)^{r+1} \cdot(r+1) \cdot F r
$$

$$
K_{c l}=A \cdot\left(\frac{k}{e}\right)^{2} \cdot \sigma \cdot T
$$

$$
\begin{aligned}
& A=\left[\frac{r+3}{r+1} \cdot \frac{F r+2}{F r+1}-\frac{(r+2)^{2}}{(r+1)^{2}} \cdot \frac{F r^{2}+1}{F r^{2}}\right] \\
& A=r+2 \text { (gaz dégénéré) } .
\end{aligned}
$$


$F r+1$ et $F r+2)$ ont été, soit recherchées dans les tables de J. Mc Dougall et E. C. Stoner [7], soit calculées par nos soins à l'aide d'une calculatrice digitale.

II. Technique expérimentale. - II.1 TeChNOLOGIE DES MATÉRIAUX. - Les différents éléments des piles (P 1) à (P 4) se présentent sous forme de pastilles cylindriques solides : diamètre 8,10 et $12 \mathrm{~mm}$; hauteur : de quelques millimètres à plusieurs centimètres suivant l'expérience considérée.

Plus particulièrement, les pastilles d'iodure d'argent et de cuivre, ainsi que de sulfure d'argent, ont été réalisées à la presse hydraulique $(7000$ bars $)$ à partir de produits en poudre.

Le sulfure cuivreux est fabriqué au laboratoire, à partir de tournures de cuivre mises en présence de vapeurs de soufre à $450^{\circ} \mathrm{C}$. Le produit obtenu est broyé, puis finement tamisé. Les pastilles de sulfure cuivreux sont alors réalisées comme précédemment.

Les pastilles de séléniure et de tellurure de cuivre sont obtenues directement par compression unilatérale (10 000 bars à $170^{\circ} \mathrm{C}$ pendant une heure) de poudres très pures de cuivre et de chalcogène intimement mélangées [9].

II. 2 Dopage ÉLECTRochimiQue et TRACÉ Des CARACTÉRISTIQUES. - L'assemblage des pastilles, permettant la réalisation des piles ( $\mathbf{P} 1$ à $\mathbf{P} 4$ ), s'effectue dans un tube de verre. Les électrodes, d'amenée du courant, sont des disques de platine. L'ensemble est comprimé à l'aide de ressorts métalliques afin d'assurer les contacts macroscopiques indispensables entre les différents éléments solides. Les piles sont placées, sous atmosphère d'argon, à l'intérieur d'un four électrique (Fig. 1a).

La force électromotrice des piles ainsi constituées dépend de la composition des chalcogénures, et possède au départ une valeur qui est fonction du mode de fabrication de ces produits. Il faut noter ici que le chalcogénure constitue toujours la cathode (pôle positif) de ces piles.

Cependant, les iodures d'argent et de cuivre ayant une conduction purement cationique [10], l'adjonction dans le circuit extérieur de pile d'un générateur électrique auxiliaire permet d'ajouter (générateur auxiliaire monté en série) ou de retrancher (montage en opposition) une certaine quantité de métal dans son chalcogénure. Seuls les ions métal traversent l'iodure, tandis que les électrons passent par le circuit extérieur où leur débit est mesuré. Ions et électrons se recombinent ensuite dans la cathode faisant varier ainsi la composition du chalcogénure, et par suite la force électromotrice de la pile.

La variation de la concentration en métal, ou encore en charges électriques, dans le chalcogénure correspondant à deux valeurs différentes de la force électromotrice est ainsi déduite de la mesure du débit électrique dans le circuit extérieur de pile. Le tracé des caractéristiques (force électromotrice - concen-

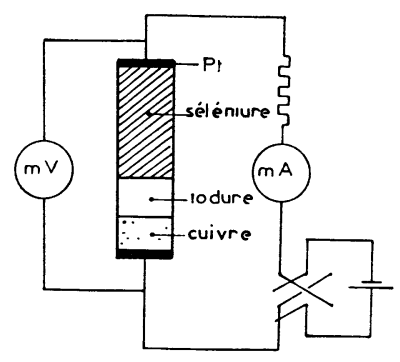

(a)

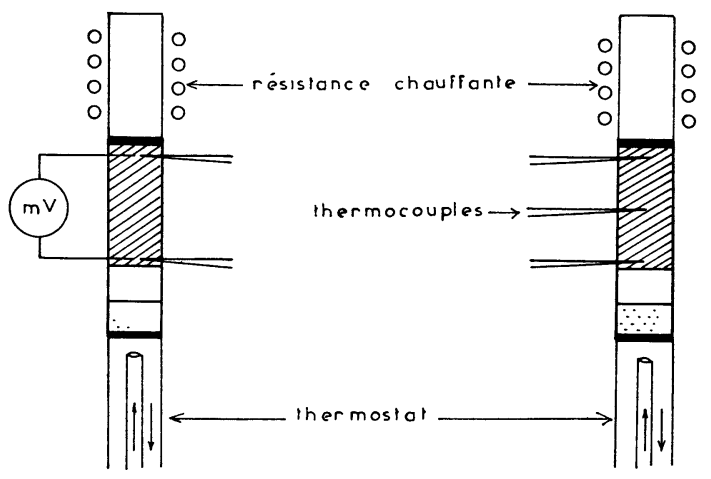

(c)
FIG. 1. - Montages expérimentaux relatifs à la détermination des propriétés thermoélectriques des chalcogénures métalliques :

$$
\begin{aligned}
& a . \text { - Dopage électrochimique } \\
& b . \text { - Conductivité électrique } \\
& c . \text { - Pouvoir thermoélectrique } \\
& d . \text { - Conductivité thermique }
\end{aligned}
$$

tration des charges électriques) n'est cependant possible que dans la mesure où l'on connaît un point de ces caractéristiques. Dans tous les cas étudiés ici, ce point peut être obtenu en considérant que la force électromotrice est nulle lorsque la concentration est maximale, c'est-à-dire lorsque le métal précipite dans son chalcogénure : le potentiel de la cathode est alors égal à celui de l'anode (métal pur).

Les caractéristiques (force électromotrice - concentration) sont représentées sur la figure 2 .

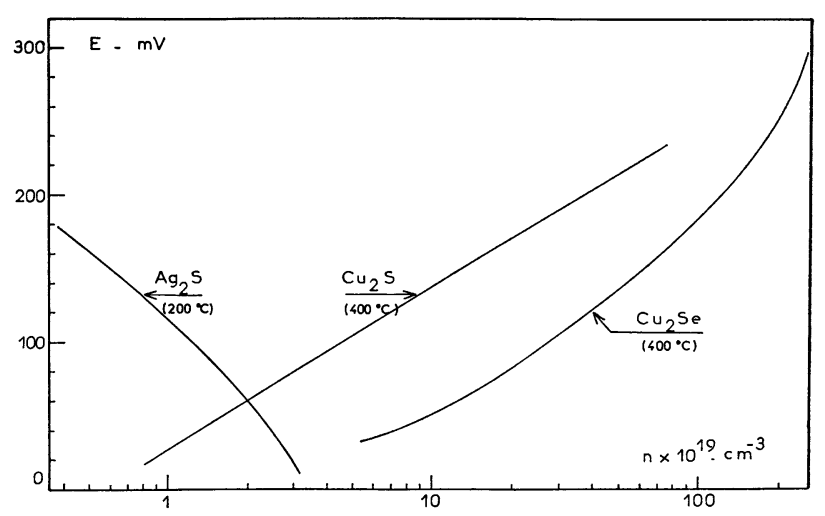

FIG. 2. - Cảractéristiques

(force électromotrice - concentration électronique). 
II. 3 MESURE DES PROPRIÉTÉS THERMOÉLECTRIQUES. Ainsi, la présence de l'iodure et du métal, à côté des chalcogénures, permet non seulement de connaître la concentration en charges électriques des thermoéléments, mais assure encore la variation de cette concentration. Pour une composition donnée des chalcogénures, repérée à l'aide d'une force électromotrice de pile, nous effectuons les différentes mesures du pouvoir thermoélectrique et des conductivités électrique et thermique. Ensuite, à l'aide du circuit extérieur de pile, nous avons la possibilité de faire varier cette composition et de suivre ainsi les variations de chaque propriété thermoélectrique dans tout le domaine de variation de la concentration électronique des thermoéléments considérés.

II.3.a Conductivité électrique (Fig. 1b). - On applique, à l'aide d'un générateur électrique, une différence de potentiel aux extrémités du chalcogénure. La conductivité électronique est alors déduite de la différence de potentiel existant entre deux électrodes constituées par des fils de platine plongeant dans l'échantillon.

Un léger gradient thermique subsiste toujours dans l'élément et donne ainsi naissance à une force thermoélectrique qui se superpose à la chute de potentiel ohmique. Afin d'éliminer ce phénomène parasite, on fait circuler successivement dans l'élément deux courants d'égale intensité, mais de sens opposés : la demi-somme arithmétique donne alors la différence de potentiel convenable.

Les résultats pratiques sont portés sur la figure 3 dans le cas du sulfure d'argent [11] et du séléniure cuivreux.

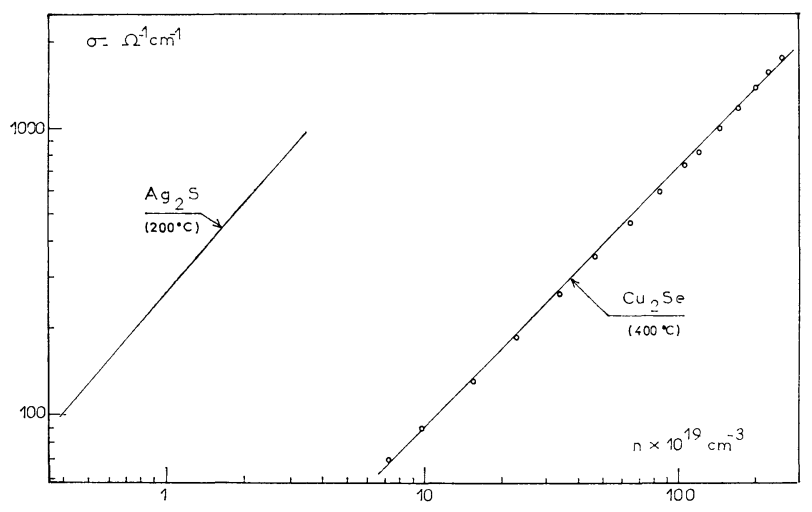

Fig. 3. - Conductivités électriques et concentrations électroniques.

II.3.b Pouvoir thermoélectrique (Fig. 1c). - Un gradient thermique, de l'ordre de quelques degrés, est établi dans le chalcogénure entre une source chaude constituée par une résistance électrique et une source froide réalisée à l'aide d'un thermostat. Ce gradient thermique est mesuré au moyen de deux thermocouples différentiels. La force thermoélectrique est repérée par l'intermédiaire de deux élec- trodes de platine situées le plus près possible des thermocouples.

Les résultats pratiques sont consignés sur la figure 4 en ce qui concerne les sulfures d'argent et de cuivre.

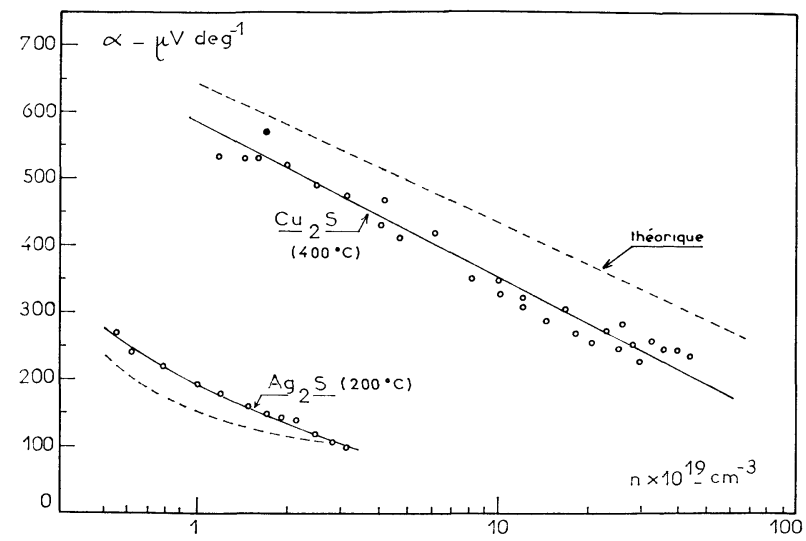

FIG. 4. - Pouvoirs thermoélectriques et concentrations électroniques.

II.3.c Conductivité thermique (Fig. 1d). - Le dispositif expérimental est le même que celui employé pour la mesure du pouvoir thermoélectrique. Un gradient thermique est établi dans le chalcogénure, tandis qu'une perte systématique de chaleur s'effectue par la surface latérale. La présence de trois thermocouples, situés à différents niveaux de l'échantillon, permet la mesure du rapport (conductivité thermique - coefficient d'échange avec le milieu extérieur) [12]. Le coefficient d'échange doit être évalué à l'aide d'une expérience indépendante où les variations de température du four et du chalcogénure sont étudiées en fonction du temps.

Les résultats expérimentaux relatifs aux conductivités thermiques des sulfures d'argent et de cuivre sont représentés sur la figure 5 .

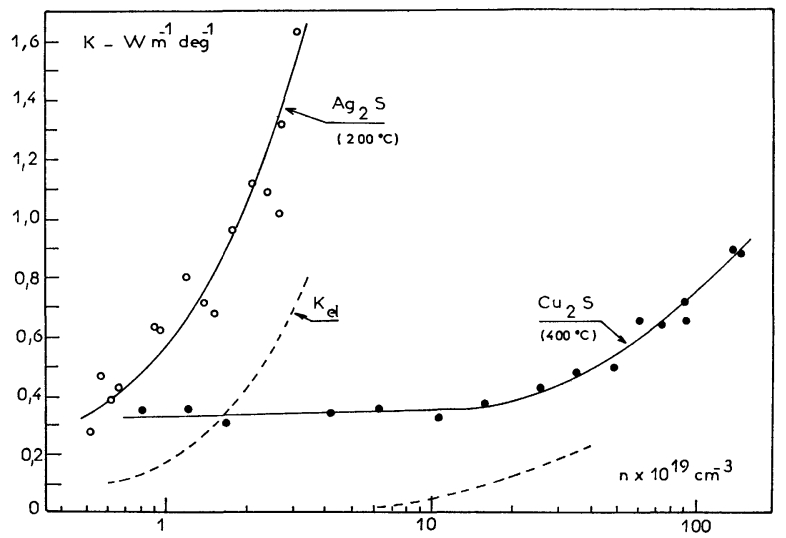

Fig. 5. - Conductivités thermiques et concentrations électroniques.

II. 4 FACTEUR DE MÉRITE. - L'ensemble des résultats expérimentaux portant sur les propriétés thermoélectriques permet, d'après l'équation (3), le calcul du facteur de mérite. 
La figure 6 montre la variation de ce facteur avec la concentration électronique dans le cas des sulfures d'argent et de cuivre. Un maximum du facteur de mérite est trouvé, en ce qui concerne le sulfure cuivreux, pour une concentration en trous électroniques positifs égale à $20 \times 10^{19} \mathrm{~cm}^{-3}$.

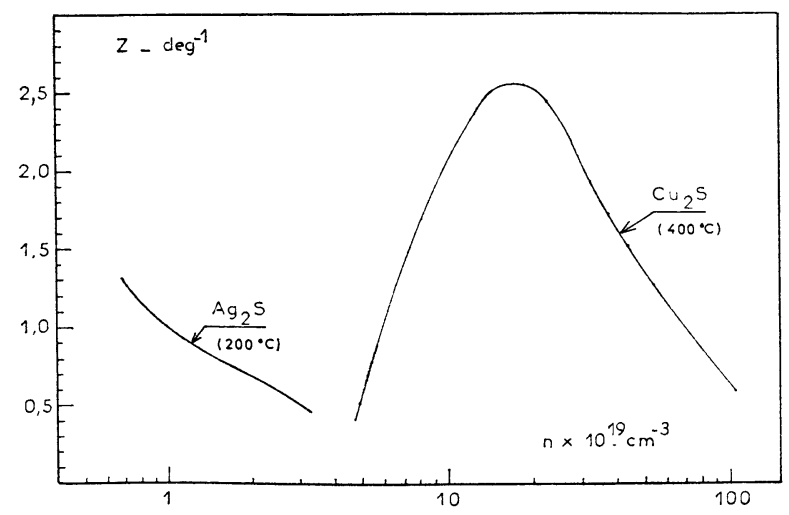

FIG. 6. - Facteurs de mérite des sulfures d'argent et de cuivre.

Bien que la concentration électronique du sulfure d'argent soit plus faible que celle du sulfure cuivreux, le caractère métallique est plus marqué dans le cas du premier sulfure. Il convient, en effet, de tenir compte, dans les réseaux ioniques, de la présence des ions à côté des charges électroniques, présence dont l'influence est très sensible sur la valeur de la masse électronique effective. Il en résulte que le gaz d'électrons est dégénéré dans le cas de sulfure d'argent (potentiel chimique des électrons positifs) alors que le gaz de trous positifs dans le sulfure cuivreux ne l'est pas (potentiel chimique des trous négatifs). Le sulfure cuivreux, par rapport au sulfure d'argent, possède ainsi des propriétés thermoélectriques de qualité supérieure.

III. Elément de thermopile utilisant les sulfures d'argent et de cuivre. - III.1 DISPOSITIF EXPÉRIMENTAL (Fig. 7). - Constituons un élément de thermopile à l'aide des deux thermoéléments suivants : sulfure d'argent (semi-conducteur de type $n$ ) et sulfure cuivreux (semi-conducteur de type $p$ ).

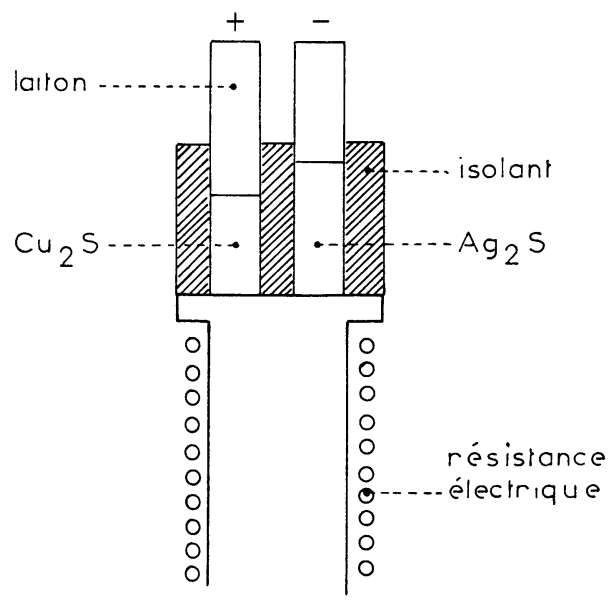

Fig. 7. - Elément de thermopile utilisant des sulfures d'argent et de cuivre.
La source primaire, fournissant la chaleur à la soudure chaude, est constituée par une résistance électrique montée sur un cylindre en laiton. L'évacuation de la chaleur à la soudure froide s'effectue par l'intermédiaire de cylindres de laiton surmontant chaque thermoélément. L'isolation des thermoéléments est assurée à l'aide de tubes de verre, de " clingérite » ou de " mulite ".

Les caractéristiques des thermoéléments sont résumées dans le tableau III.

\section{TABleaU III}

Caractéristiques des thermoéléments

Thermoélément

$\begin{array}{cc}\begin{array}{c}\text { Sulfure } \\ \text { d'argent }\end{array} & \begin{array}{c}\text { Sulfure } \\ \text { de cuivre }\end{array} \\ - & - \\ 12 \mathrm{~mm} & 12 \mathrm{~mm} \\ 11,5 \mathrm{~mm} & 7,5 \mathrm{~mm} \\ 500^{\circ} \mathrm{C} & 500^{\circ} \mathrm{C} \\ 275^{\circ} \mathrm{C} & 275^{\circ} \mathrm{C}\end{array}$

Diamètre

Hauteur

Soudure chaude

Soudure froide

Composition

correspondant au maximum du facteur de mérite d'après la figure $\mathrm{n}^{0} 6$

Les hauteurs sont choisies de manière à obtenir des gradients thermiques égaux aux extrémités de chaque élément.

III.2 Résultats eXpérimentaux. - Pour un gradient thermique de l'ordre de $225^{\circ} \mathrm{C}$, l'élément de thermopile est assujetti à débiter sur une résistance de charge de $1 \Omega$ (résistance sensiblement égale à la résistance interne de la thermopile). La force électromotrice est alors de $100 \mathrm{mV}$ et le courant débité de l'ordre de $60 \mathrm{~mA}$.

La figure 8 montre la variation de la force électromotrice et de l'intensité débitée par la thermopile pour différents gradients $\left(T_{\mathrm{c}}-T_{\mathrm{f}}\right)$ appliqués aux bornes des thermoéléments et obtenus en diminuant la température de la soudure chaude $T_{\mathrm{c}}$.

Pour de faibles gradients thermiques, inférieurs à $100^{\circ} \mathrm{C}$, la température de la soudure froide du sulfure d'argent se trouve être inférieure à $175^{\circ} \mathrm{C}$. A cette température il se produit un changement de phase de ce composé $[13,3]$ : la conductivité électrique diminue brusquement et fortement [14] tandis que le pouvoir thermoélectrique augmente [15]. Il en résulte une diminution de l'intensité débitée et une augmentation de la force électromotrice de la thermopile. Ces faits sont bien marqués sur la figure 8 .

III. 3 REMARQUE SUR L'EMPLOI DES SULFURES D'ARGENT ET DE CUIVRE EN TANT QUE THERMOÉLÉMENTS. L'observation prolongée du fonctionnement de la thermopile dénote un manque de stabilité des sulfures d'argent et de cuivre en tant que thermoélément.

En effet, sous l'action d'un gradient thermique, il existe un gradient d'activité du métal dans son sulfure qui provoque la diffusion de ce métal d'une soudure à l'autre. Dans le cas du sulfure d'argent, de l'argent 


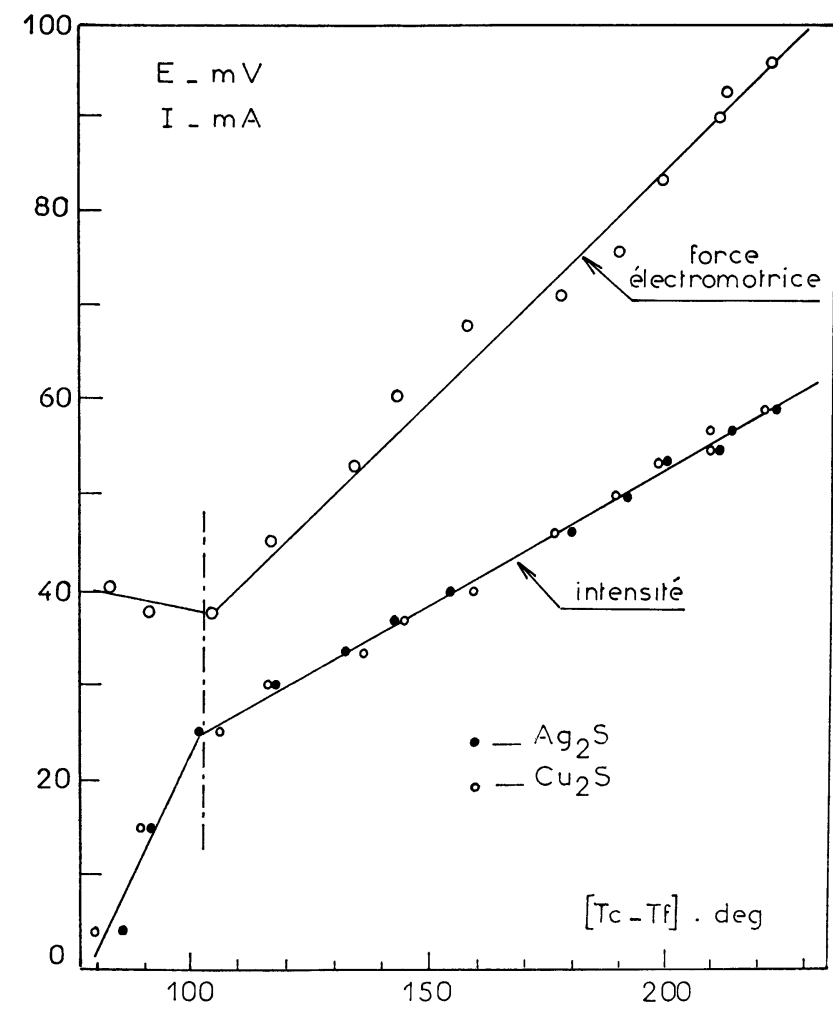

Fig. 8. - Intensité débitée et force électromotrice de la thermopile en fonction du gradient thermique aux extrémités des thermoéléments.

métallique, sous forme d'ions et d'électrons, émigre de la soudure chaude à la soudure froide [16]. De plus, les différences de tension de vapeurs de soufre au-dessus du sulfure provoquent une migration en phase vapeur.

On note ainsi, après quelques heures de fonctionnement, l'apparition d'argent métallique au niveau de la soudure froide du sulfure d'argent, le soufre s'évaporant par ailleurs. Le phénomène est inversé dans le cas du sulfure cuivreux : du cuivre métallique apparaît à la soudure chaude, et par suite des difficultés technologiques relatives à la mise sous atmosphère inerte des thermoéléments, on note la trans- formation de ce cuivre en oxyde de cuivre, puis en sulfate sous l'action de l'air. Ces derniers produits provoquent généralement l'arrêt en débit de la thermopile.

Conclusion. - Dans les montages expérimentaux que nous avons réalisés en vue de l'étude des propriétés thermoélectriques des chalcogénures métalliques, nous avons toujours associé à ces chalcogénures les iodures et métaux correspondants : la mesure de la force électromotrice de la pile ainsi constituée donne immédiatement la composition $\mathrm{du}$ chalcogénure. Par action sur les caractéristiques du circuit extérieur de pile, on est en mesure de choisir cette composition et de la faire évoluer dans le sens désiré. On peut ainsi, d'une manière continue, suivre la variation d'une de ces propriétés thermoélectriques en fonction du dopage du chalcogénure. Les résultats expérimentaux sont confrontés, chaque fois, à ceux que laisse présager la statistique de Fermi-Dirac, appliquée au gaz électronique.

La réalisation et l'étude d'un élément de thermopile utilisant les sulfures d'argent et de cuivre comme thermoéléments montrent que, si des résultats convenables ont été obtenus quant à la force électromotrice et au courant électrique débité, il semble que l'emploi de ce type de chalcogénures soit limité par leur manque de stabilité sous l'action de forts gradients thermiques.

La présente étude suggère donc l'utilisation de chalcogénures où les phénomènes de migration ionique soient partiellement exclus et où les tensions de vapeurs des éléments soient les plus faibles possibles. Les séléniures et tellurures, composés présentant un caractère métallique plus marqué, paraissent donc plus aptes à la réalisation de thermopiles. Il est enfin possible de dresser l'inventaire des difficultés de construction d'une thermopile : mise sous atmosphère inerte des éléments, oxydation des plaques métalliques de connexion, apport de chaleur de la source primaire à la soudure chaude, isolation des thermoéléments, évacuation de la chaleur à la soudure froide ; problèmes qui ne sont, à l'heure actuelle, que partiellement résolus.

\section{Bibliographie}

[1] Miyatani (S.) et Toyota (Y.), J. of Phys. Soc. of Japan, 1967, 23, 1.

[2] Routie (R.) et Mahenc (J.), C. R. Acad. Sc. Paris, 1968, série C, 267, 1937-38.

[3] Wagner (C.), J. Chem. Phys., 1953, 21, 1819.

[4] Wagner (J. B.) et Wagner (C.), J. Chem. Phys., 1957, 26, 1602.

[5] Lorentz (G.) et Wagner (C.), J. Chem. Phys., 1957, 26, 1607.

[6] Routie (R.) et MAHenc (J.), J. de Chimie Phys., 1968, $65,1331$.

[7] Mc Dougall (J.) et Stoner (E. C.), Trans. Roy. Soc., London, 1938, A 237, 67.

[8] IofFE (A. F.), Semiconductor thermoelements, Infosearch, Ltd, London, 1967.
[9] Mole (R.) et Hocart (R.), Bull. Soc. Chim. de France, 1954, 8, 977.

[10] Wagner (J. B.) et Wagner (C.), J. Chem. Phys., 1957, 26, 1597

[11] Miyatani (S.), J. of Phys. Soc. of Japon, 1955, 10, 786.

[12] FABRY (C.), Propagation de la chaleur, Armand Colin, 1942.

[13] KraceK (F. C.), Trans. Ann. Geophys. Union, 1946, 27, 364.

[14] Hebs (M. H.), J. Chem. Phys., 1959, 20, 185.

[15] Routie (R.) et Mahenc (J.), J. de Chimie Phys., 1969, 66, 834.

[16] RickeRT (H.) et WAGNER (C.), Berichte der Bunsengesellschaft für phys. chem., 1963, 67, 621-29. 